Research Article

\title{
Cartilage Injury after Patellar Dislocation Surgery and Evaluation of the Effect of Platelet-Rich Plasma Treatment by Algebraic Reconstruction Techniques Based MRI
}

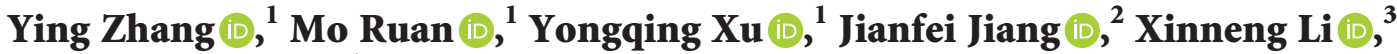 \\ and Hongbo Tan $\mathbb{1}^{1}$ \\ ${ }^{1}$ Department of Orthopedics, No. 920 Hospital of the Joint Logistic Support Force of the PLA of China, Kunming 652000, \\ Yunnan, China \\ ${ }^{2}$ Kunming Gaoyao Medical Imaging Diagnostic Center, Kunming 650504, Yunnan, China \\ ${ }^{3}$ Department of Orthopedics, Zhenkang People's Hospital, Lincang 677799, Yunnan, China
}

Correspondence should be addressed to Hongbo Tan; 201422030275@mail.bnu.edu.cn

Received 31 August 2021; Revised 14 October 2021; Accepted 15 October 2021; Published 31 October 2021

Academic Editor: Gustavo Ramirez

Copyright (c) 2021 Ying Zhang et al. This is an open access article distributed under the Creative Commons Attribution License, which permits unrestricted use, distribution, and reproduction in any medium, provided the original work is properly cited.

\begin{abstract}
The study focused on the application value of Algebraic Reconstruction Techniques (ART) based magnetic resonance imaging (MRI) in the diagnosis of cartilage injury in patients with patellar dislocation, as well as the treatment effect of platelet-rich plasma (PRP). 50 patients with patellar dislocation admitted to the hospital were selected as the research subjects, and they were randomly divided into the experimental group (lateral retinaculum plasty and PRP treatment) and the control group (lateral retinaculum plasty), with 25 cases in each group. The ART-based MRI technology was used to judge the recovery of patients after surgery and analyze the differences in clinical data between the two groups. The results showed that the running speed under ART algorithm was significantly faster than the traditional Joseph algorithm and Siddon algorithm, and the image reconstruction effect was better. The visual analog scale (VAS) scores of preoperative and postoperative pain and the Hospital for Special Surgery (HSS) score in the experimental group were better than those of the control group $(P<0.05)$. In conclusion, the ART-based MRI technology can clearly show cartilage injury and the PRP treatment can effectively relieve postoperative pain, with reliable curative effects and simple operations.
\end{abstract}

\section{Introduction}

Patella, also known as the kneecap, is in front of the knee joint and forms the patellofemoral joint together with the femur. In recent years, with the improvement of people's quality of life, fitness exercise has gradually become popular. However, because of various reasons, such as incorrect fitness methods, the incidence of knee joint injuries has also increased year by year. Among them, patellar dislocation is common. Generally speaking, patella dislocation is when the knee joint is bent, the patella deviates from the groove of the femur. Patella dislocation mainly occurs in adolescents and sports enthusiasts, and it is also common in female groups. The pathogenic factors are mostly congenital patella position, shape, or femoral trochlear dysplasia. According to different causes, patella dislocation can be divided into acute traumatic patella dislocation, recurrent patella dislocation, and habitual patella dislocation.

Platelet-rich plasma (PRP) refers to a liquid whose platelet concentration is significantly higher than the normal value, and it is often used in the treatment of bone and tendon injuries. A variety of growth factors and mediators contained in platelets contribute to the body's natural healing process [1]. PRP preparations can be further refined into leukocyte-rich PRP preparations and leukocyte-poor preparations. The difference between the two is whether the concentration of neutrophils is higher than the baseline concentration [2]. Nevertheless, because of the distinct types of operations and equipment 
used during the preparation process, the specific components of PRP preparations may differ. As a result, the clinical efficacy of PRP is difficult to evaluate. Studies have shown that, after two years of PRP injection, the experimental group shows a significant effect at 12 weeks of follow-up, but at 26 weeks and above, the difference between the two groups is no longer obvious, which indicates that the efficacy of PRP is not lasting [3]. Although there has been clinical research on the application of PRP alone, there are few studies on the combined treatment of PRP with another preparation.

Magnetic resonance imaging (MRI) images have high resolution for soft tissue and will not cause trauma, which is a popular method to examine knee joint diseases [4]. The Algebraic Reconstruction Technique (ART) is the main part of the iterative algorithm. The basic idea is to disperse the image into a series of pixels, establish a series of equations to be solved based on the projection data, and obtain a new reconstructed image $[5,6]$. In this study, it was explored whether MRI based on the ART algorithm can effectively diagnose cartilage injury in patients after patellar dislocation, and the effect of PRP on the postoperative cartilage injury was analyzed.

\section{Materials and Methods}

2.1. Research Subjects. In the study, 50 patients admitted to the hospital for treatment of patellar dislocation between January 2018 and February 2020 were selected as research subjects, and their clinical data were collected. Of the 50 subjects, there were 12 males and 38 females, aged $20-55$ years old. They were randomly divided into the experimental group and the control group according to different treatment methods, with 25 in each. The experimental group was treated with lateral retinaculum plasty combined with PRP (Guizhou Tedia Medical Instruments Co., Ltd, China). The control group was treated with lateral retinaculum plasty alone.

The subjects were selected as per the following inclusion criteria: (I) diagnosed with patellar dislocation, (II) with complete clinical data, (III) aged less than 60 years, and (IV) the family members and the patient himself signed an informed consent form voluntarily.

The exclusion criteria were as follows: (I) nonpatellar dislocation patients, (II) patients with mental illness, and (III) patients who did not sign the informed consent or withdrew from the experiment halfway.

2.2. MRI Examination. In this study, the MRI workstation is used for measurement. The thickness can be directly measured on the FS-3D-FISP sequence imaging, and the measurement method refers to relevant literature reports. The T2 value is obtained using T2WI sagittal double echo imaging to obtain the T2 quantified map first and then measured at the lesion. Two physicians with clinical experience independently measured the thickness of the postoperative cartilage in the FS-3D-FISP sequence, and the means of the thickness and the $\mathrm{T} 2$ value were taken, respectively.
2.3. ART Algorithm. The ART algorithm is expressed as follows:

$$
f_{j}^{(k+1)}=f_{j}^{(k)}+\lambda^{(k)} \frac{g_{i}-\sum_{j-1}^{N} w_{i j} f_{n}}{\sum_{j-1}^{N} w_{i j}^{2}} w_{i j},
$$

where $k$ is the number of iterations, $f_{j}^{(k)}$ is the pixel value numbered $j$ after the $k$ th iteration, $\lambda^{(k)}$ is the iteration factor at the $k$ th iteration, and $w_{i j}$ is the projection of the $j$ th pixel on the $i$ th ray (weight factor).

The algorithm uses line-by-line correction, and the correction value is the difference between the projection value and the real projection. Each time the current image is updated, all rays are corrected once under the current projection angle, and then the correction under the next projection angle is performed. When all the angles are involved, it means that an iteration is completed. The specific steps are as follows: (I) the image to be measured is dispersed and assigned an initial value; (II) the theoretical projection value $g_{i}=\sum_{j=1}^{N} w_{i f} f_{j}^{(k)}$ of the $i$ th ray is calculated; (III) $\chi_{i j}=\Delta_{i} w_{i j} / \sum_{j} w_{i j}^{2}$, the current correction value is calculated; (IV) the correction value $f_{j}=f_{j}^{(k)}+\chi_{i j}$ is used to calculate the $j$ th pixel; (V) steps (II)-(V) are repeated for other rays in the current projection direction; (VI) the above steps are repeated in other directions to complete an iteration; and (VII) the final result is regarded as the initial value of the next iteration, and the previous steps are repeated until the equations converge.

2.4. Simulation Experiment. Simulation environment: VS2017 under WIN10 system, the hardware environment is Intel core 6 core processor, the CPU frequency is $3.60 \mathrm{GHz}$, and the computer memory is $64 \mathrm{G}$.

Network parameters: the image resolution is 512512, and the pixel $\times$ size is $0.023 \mathrm{~mm}^{2}$.

The Joseph algorithm and Siddon algorithm are introduced to compare with the algorithm in the study in terms of the numerical value and reconstruction speed.

Numerical analysis involves three parameters of normalized root mean square distance measurement value $(d)$, normalized average absolute distance measurement value $(r)$, and image similarity coefficient measurement value $(\varepsilon)$. A smaller value of $d$ and $r$ means that the measured image is closer to the reference image, and a larger value of $\varepsilon$ means that the measured image is closer to the reference image. They are expressed as follows:

$$
\begin{aligned}
& d=\left(\frac{\sum_{i=1}^{N}\left(x_{i}-x_{i}^{*}\right)^{2}}{\sum_{i=1}^{N}\left(x_{i}-\bar{x}\right)^{2}}\right)^{1 / 2}, \\
& r=\frac{\sum_{i=1}^{N}\left|x_{i}-x_{i}^{*}\right|}{\sum_{i=1}^{N} x_{i}}, \\
& \varepsilon=\frac{\sum_{i=1}^{N}(x-\bar{x})\left(x_{i}^{*}-\overline{x^{*}}\right)}{\left[\sum_{i=1}^{N}(x-\bar{x})^{2} \sum_{i=1}^{N}\left(x_{i}^{*}-\overline{x^{*}}\right)^{2}\right]^{1 / 2}},
\end{aligned}
$$

where $N$ is the total number of pixels, $x_{i}$ is the gray value of the $i$ th pixel of the reference image, $x_{i}^{*}$ is the gray value of the 
$i$ th pixel of the measured image, $\bar{x}$ is the average gray value of the reference image, and $\overline{x^{*}}$ is the average gray value of the measured image.

2.5. Surgical Methods. All patients accepted lateral retinaculum plasty for patella reduction. At the same time, the experimental group received PRP treatment after surgery and the control group received conservative treatment.

The specific surgical steps were as follows. The two groups shared the same group of physicians. The patient underwent combined spinal-epidural anesthesia in a supine position. Then, the affected lower limbs were routinely disinfected and draped. After defining the position of the patella, the deep fascia layer, lateral retinaculum layer, and joint capsule were separated from the loose lateral retinaculum. Next, according to the patella track, the free end of the separator was sutured to the middle of the outer edge of the patella or the upper third of the patella. Similarly, a suitable soft tissue strip can be trimmed from the loose inner support belt, and the soft tissue strip can be sutured with the loosened lateral joint capsule.

The experimental group received PRP treatment after lateral retinaculum plasty. The preparation of PRP was as follows. $10 \mathrm{~mL}$ venous blood was drawn before surgery and centrifuged at $2000 \mathrm{r} / \mathrm{min}$ for 10 minutes at $4^{\circ} \mathrm{C}$. Then, the supernatant, as well as the white blood cell and platelet layer, was transferred to another blood collection tube. Again, centrifugation at $2000 \mathrm{r} / \mathrm{min}$ was performed for 10 minutes at $4^{\circ} \mathrm{C}$, and then $2 \mathrm{~mL}$ of supernatant and white blood cell and platelet layer were collected. After suspension, calcium chloride was added to activate platelets to produce PRP. The knee joint of the affected limb was routinely disinfected twice. With the intersection of the upper edge of the patella with space between the patella and the knee joint as the insertion point, $2 \mathrm{~mL}$ PRP was injected into the joint cavity, and then the knee passively flexed and extended 10 times after the injection. The injection site should avoid contact with water within 24 hours after injection. Ice compress was recommended for patients with knee pain, and nonsteroidal anti-inflammatory analgesics should be taken orally if the VAS score was more than 5 points. PRP was injected once every 7-10 days for a total of 6 injections.

2.6. Evaluation Criteria. Different algorithms were compared for normalized root mean square distance measurement value $(d)$, normalized average absolute distance measurement value $(r)$, image similarity coefficient measurement value $(\varepsilon)$, and reconstruction time. The postoperative pain in the affected area is evaluated by the visual analog scale (VAS). The total score is 10 points, and a higher score indicates that the pain is more unbearable. After the surgery, the patient's recovery was evaluated according to five aspects of pain, joint flexibility, function, knee flexion, and muscle strength as per Hospital for Special Surgery (HSS).
2.7. Statistics. The experimental data were processed using SPSS19.0, the measurement data were represented by the mean \pm standard deviation $(\bar{x} \pm s)$, the count data were represented by $n$, and the comparison between groups adopted the $t$-test or $\chi^{2}$ test. $P<0.05$ was the threshold for significance.

\section{Results}

3.1. The Basic Data. According to the inclusion and exclusion criteria, 50 patients who were diagnosed with patellar dislocation were selected, and their clinical data were collected. There were 23 males and 27 females, aged between 20 and 55 years, with an average age of $45.32 \pm 6.23$ years. There were 23 cases with patellar dislocation on the left knee and 27 cases on the right knee. The preoperative MRI examination showed varying degrees of femoral condyle cartilage injury. As per the Outerbridge cartilage classification standard, there were 9 cases of I grade, 13 cases of II grade, 17 cases of III grade, and 11 cases of IV grade (Figure 1).

It was noted from Figures 1 and 2 that there was no significant difference in the clinical data of the two groups of patients, and they were comparable.

3.2. Simulation Experiment Results. It was evident from Figure 3 that, in terms of image $\varepsilon$ similarity, the ART algorithm was superior to the other two. At the same time, its $r$ value and $d$ value were smaller than the Siddon algorithm.

It was evident from Figure 4 that, in terms of the time to calculate the projection coefficients, the ART algorithm took much less time than the other two. It was more than three times faster than the Joseph algorithm and five times faster than the Siddon algorithm. Also, the single iteration of ART algorithm was also less time-consuming than the other two, which was more than 2 times faster than the Joseph algorithm and nearly three times faster than the Siddon algorithm.

3.3. Clinical Observation Indexes. It was noted from Figures 5 and 6 that, before surgery, the VAS score in the experimental group was $7.54 \pm 0.42$ points and that in the control group was $7.13 \pm 0.84$ points, showing little difference. After surgery, the VAS scores of the two groups of patients were significantly reduced, but the decline in the experimental group was more obvious, showing statistical differences $(P<0.05)$. Before surgery, the HSS score in the experimental group was $47.54 \pm 5.42$ points and that in the control group was $48.12 \pm 5.46$ points, showing significant differences. After surgery, the HSS scores of the two groups had improved, but the improvement in the experimental group was more obvious, showing statistical differences $(P<0.05)$.

3.4. Typical Case. A male patient aged 45 in the experimental group was selected. The preoperative examination showed patellar dislocation, with wear of cartilage and edema of femoral bone marrow (Figure 7). 


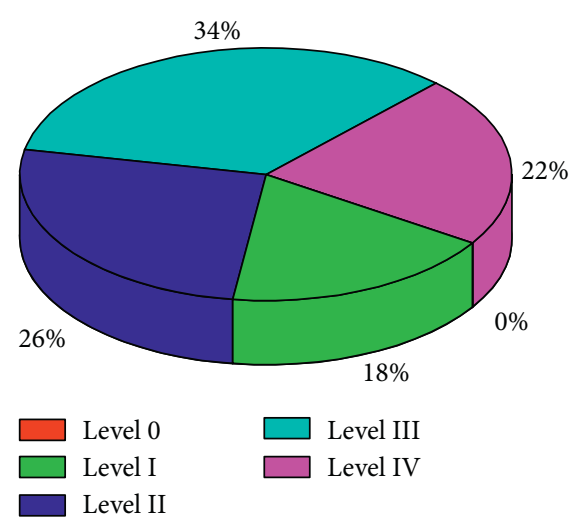

FIGURE 1: Degrees of cartilage injury in patients before surgery.

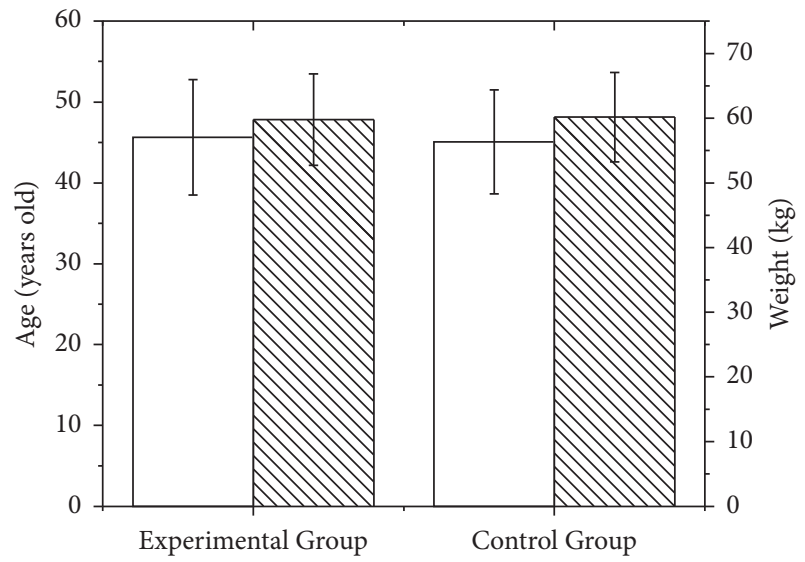

FIgURE 2: Age and weight statistics of patients in the experimental group and the control group.

Six months after the retinaculum plasty and PRP treatment, cartilage thickness returned to normal, as shown in Figure 8.

\section{Discussion}

The knee joint is a complex motion joint. In addition to the function of carrying and distributing the load, cartilage tissue also plays a role in reducing the friction of the joint surface and stabilizing the whole body. The cartilage of the knee joint is a unique connective tissue composed mainly of 99\% extracellular matrix and 1\% hyaline cartilage. These cartilage cells can secrete matrix [7], and the collagen fiber in the outer matrix can maintain the tensile strength and rigidity of the entire cartilage tissue, as well as disperse the external stress on the knee joint [8]. The proteoglycan molecules in the matrix are related to the ductility and compressibility of cartilage tissue. Because of the poor regeneration ability of normal chondrocytes and the low tolerance after regeneration, cartilage injury is difficult to repair [9]. Therefore, how to efficiently repair articular cartilage injury has been a hot spot [10].

Studies have shown that PRP contains a variety of growth factors, such as FGF, TGF- $\beta$, and PF4 [11]. These growth factors can activate platelets to promote tissue

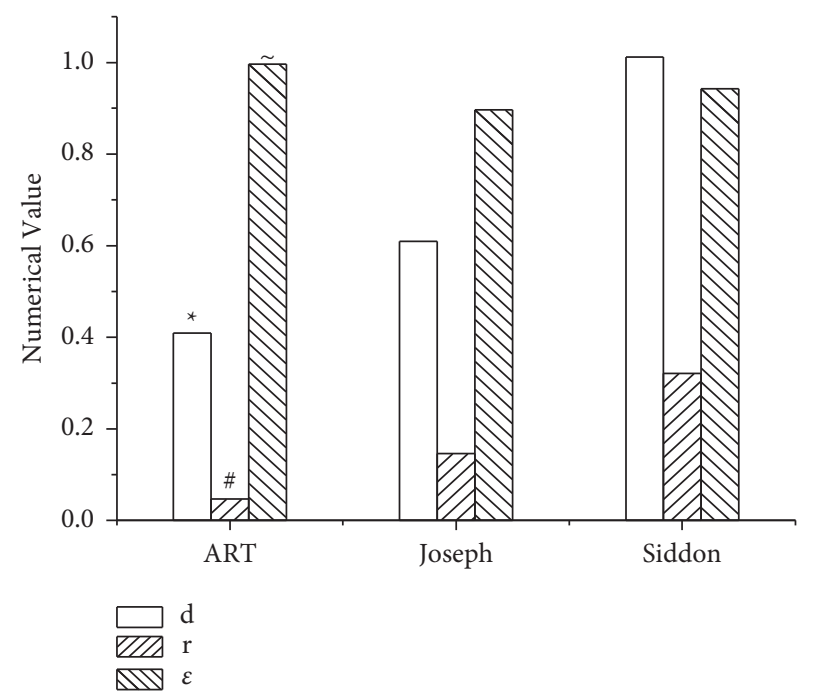

FIGURE 3: Numerical analysis of reconstructed images generated by different projection algorithms. Note. $d$ : the normalized root mean square distance measurement value; $r$ : the normalized average absolute distance measurement value; $\varepsilon$ : the image similarity coefficient measurement value; ${ }^{*}, \#$, and $\sim$ : significant differences versus the Joseph algorithm and Siddon algorithm $(P<0.05)$.

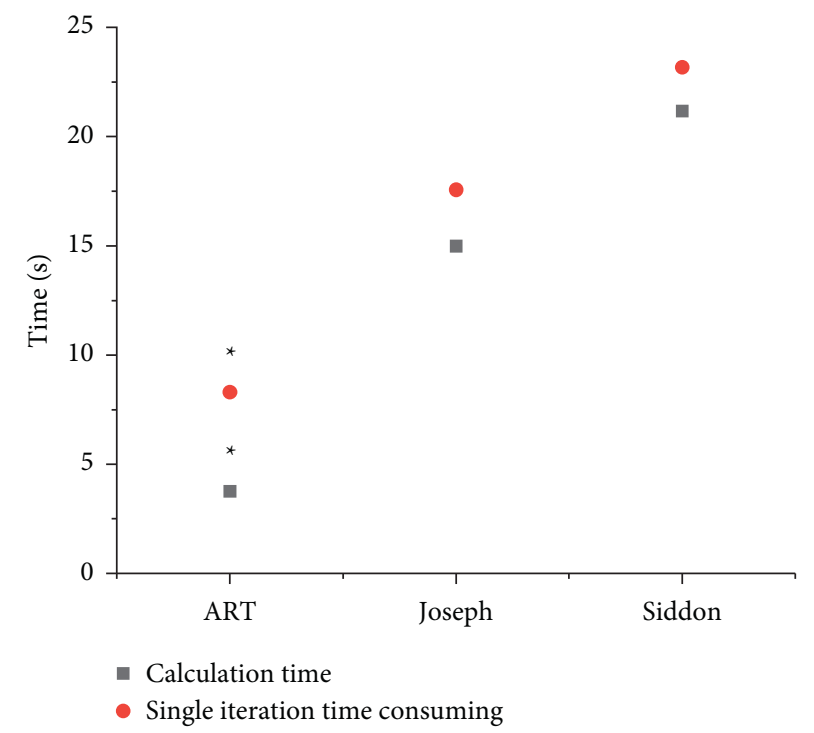

FIgure 4: The calculation speed for different algorithms takes time. * Significant differences in the running time and the time for a single iteration versus the Joseph algorithm and Siddon algorithm $(P<0.05)$.

healing [12]. The cartilage tissue of the knee joint lacks repair factors, and injecting PRP into the injured area of the knee joint is a perfect solution to this problem [13].

The treatment of cartilage injury also requires an accurate judgment of the injury. Clinically, the evaluation is mostly based on the clinical symptoms before and after the operation, but subjective judgments of the doctor are not accurate enough $[14,15]$. MRI examination can make up for the shortcomings, and it will not cause trauma like arthroscopy [16]. 


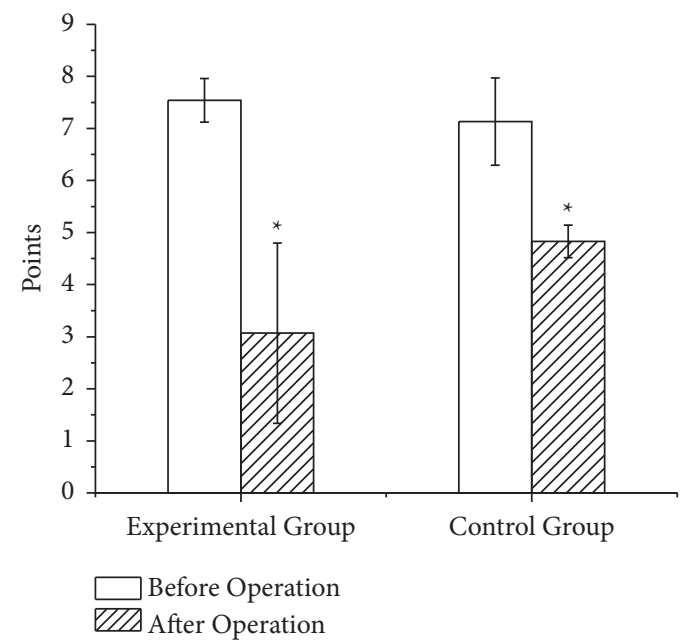

FIGURE 5: VAS scores of patients before and after surgery. ${ }^{*}$ The postoperative VAS score of the control group was statistically different compared with the experimental group $(P<0.05)$.

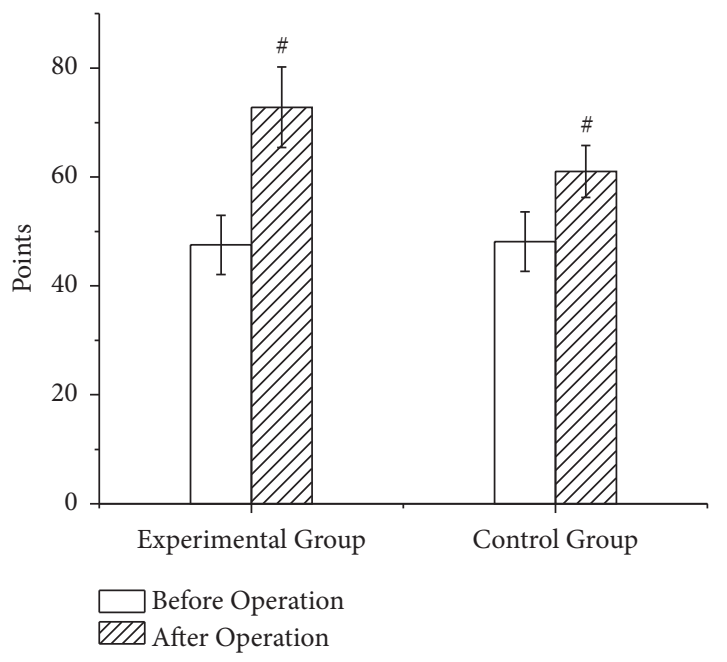

FIGURE 6: HSS score before and after surgery. ${ }^{\#}$ The postoperative HSS score of the control group was statistically different compared with the experimental group $(P<0.05)$.

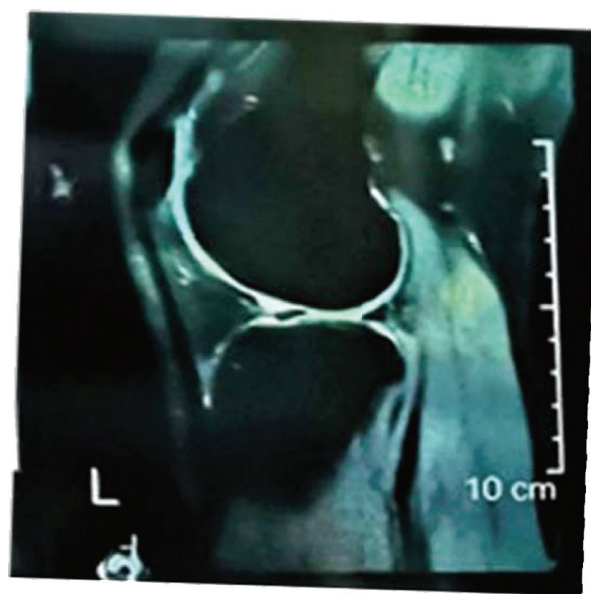

FIgURE 7: Preoperative MRI image. 


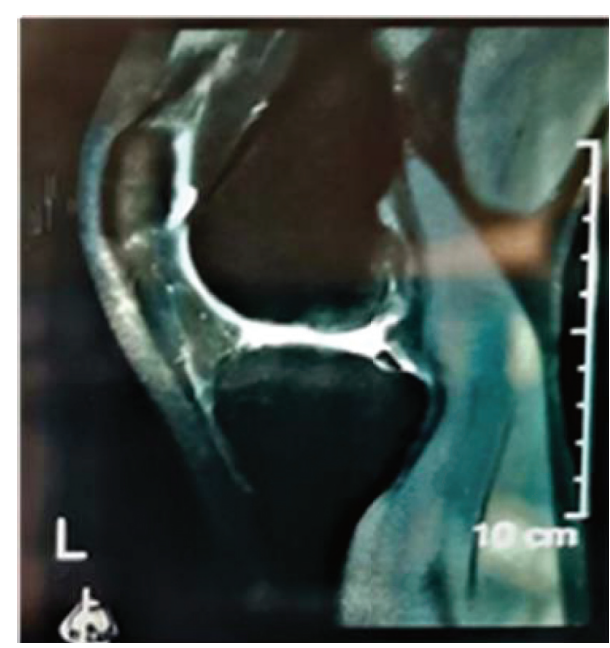

FIgURe 8: Postoperative MRI image.

The ART algorithm in the study not only guaranteed the accuracy in calculating projection coefficients but also was nearly 4 times faster than the traditional algorithm in calculation [17].

The MRI examination has good spatial resolution [18], can detect tiny cartilage damage [19], displays changes in cartilage signal intensity [20], and clearly distinguishes between switch articular cartilage and cartilage subchondral bone and joint fluid. According to the comparison of preoperative and postoperative MRI images, the cartilage thickness of the experimental group increased, and the increase was more obvious versus the control group. Of the 25 experimental group patients, 23 cases had a decreased cartilage T2 value, proving the effectiveness of the PRP treatment.

In this study, 50 patients diagnosed with patella dislocation received lateral retinaculum plasty. Additionally, 25 patients in the experimental group received PRP treatment, and the remaining 25 in the control group received the conservative treatment. It was found that knee joint function scores of patients who received PRP treatment improved more significantly, and there were no serious complications after surgery, confirming the feasibility of the treatment plan, which not only relieved postoperative pain but also sped up the recovery.

\section{Conclusion}

In this study, 50 patients diagnosed with patella dislocation received lateral retinaculum plasty. Additionally, 25 patients in the experimental group received PRP treatment and the remaining 25 in the control group received the conservative treatment. In the meanwhile, simulation experiments were carried out for the ART algorithm. It was found that the MRI examination based on the ART algorithm was faster and the images were clearer, which provided good objective conditions for postoperative follow-up observation. Lateral retinaculum plasty combined with PRP treatment demonstrated significant therapeutic effects in cartilage damage caused by patellar dislocation. However, some limitations in the study should be noted. The sample size is small, which will reduce the power of the study. In the follow-up, an expanded sample size is necessary to strengthen the findings of the study. In conclusion, ART-based MRI technology can clearly show cartilage injury and the PRP treatment can effectively relieve postoperative pain, with reliable curative effects and simple operations. The results of this study provide a reference for subsequent related treatment.

\section{Data Availability}

The data used to support the findings of this study are available from the corresponding author upon request.

\section{Conflicts of Interest}

The authors declare no conflicts of interest.

\section{Acknowledgments}

This work was supported by the National Key Research and Development Plan (Project no. 2017YFC1103904) and Science and Technology Talents and Platform Program (Project no. 2018HB001).

\section{References}

[1] B. O'Connell, N. M. Wragg, and S. L. Wilson, "The use of PRP injections in the management of knee osteoarthritis," Cell and Tissue Research, vol. 376, no. 2, pp. 143-152, 2019, Epub 2019 Feb 13. PMID: 30758709.

[2] K. Su, Y. Bai, J. Wang, H. Zhang, H. Liu, and S. Ma, "Comparison of hyaluronic acid and PRP intra-articular injection with combined intra-articular and intraosseous PRP injections to treat patients with knee osteoarthritis," Clinical Rheumatology, vol. 37, no. 5, pp. 1341-1350, 2018, Epub 2018 Jan 31. PMID: 29388085.

[3] C. Guillibert, C. Charpin, M. Raffray et al., "Single injection of high volume of autologous pure prp provides a significant improvement in knee osteoarthritis: a prospective routine care study," International Journal of Molecular Sciences, vol. 20, no. 6, p. 1327, 2019, PMID: 30884774; PMCID: PMC6472196.

[4] R. Burchard, H. Huflage, C. Soost, O. Richter, B. Bouillon, and J. A. Graw, "Efficiency of platelet-rich plasma therapy in knee osteoarthritis does not depend on level of cartilage damage," Journal of Orthopaedic Surgery and Research, vol. 14, no. 1, p. 153, 2019, PMID: 31126348; PMCID: PMC6534904.

[5] W. J. Shi, F. P. Tjoumakaris, M. Lendner, and K. B. Freedman, "Biologic injections for osteoarthritis and articular cartilage damage: can we modify disease?" The Physician and Sportsmedicine, vol. 45, no. 3, pp. 203-223, 2017, Epub 2017 Jul 28. PMID: 28719231.

[6] Z. Lv, D. Chen, R. Lou, and Q. Wang, "Intelligent edge computing based on machine learning for smart city," Future Generation Computer Systems, vol. 115, no. 1, pp. 90-99, 2021, ISSN 0167-739X.

[7] B. L. Walters, D. A. Porter, S. J. Hobart et al., "Effect of intraoperative platelet-rich plasma treatment on postoperative donor site knee pain in patellar tendon autograft anterior cruciate ligament reconstruction: a double-blind randomized controlled trial," The American Journal of Sports Medicine, 
vol. 46, no. 8, pp. 1827-1835, 2018, Epub 2018 May 9. PMID: 29741923.

[8] H. Zhang, S. Chen, M. Qiu, A. Zhou, W. Yan, and J. Zhang, "Lateral meniscus allograft transplantation with platelet-rich plasma injections: a minimum two-year follow-up study," The Knee, vol. 25, no. 4, pp. 568-576, 2018, Epub 2018 Apr 24. PMID: 29699841

[9] J. Pak, J. H. Lee, N. J. Pak et al., "Clinical protocol of producing adipose tissue-derived stromal vascular fraction for potential cartilage regeneration," Journal of Visualized Experiments, vol. 139, 2018, Article ID 58363, PMID: 30320755; PMCID: PMC6235368.

[10] T. Southworth, N. Naveen, T. Tauro, N. Leong, and B. Cole, "The use of platelet-rich plasma in symptomatic knee osteoarthritis," Journal of Knee Surgery, vol. 32, no. 1, pp. 037-045, 2019, Epub 2018 Nov 13. PMID: 30423591.

[11] Y.-C. Hsu, C.-K. You, C.-L. Chou, and W.-T. Wu, "Nonoperative choice of anterior cruciate ligament partial tear: ultrasound-guided platelet-rich plasma injection," Journal of Medical Ultrasound, vol. 27, no. 3, pp. 148-150, 2019, PMID: 31867179; PMCID: PMC6905270.

[12] H. A. Oliver, C. C. Bozynski, C. R. Cook et al., "Enhanced subchondroplasty treatment for post-traumatic cartilage and subchondral bone marrow lesions in a canine model," Journal of Orthopaedic Research, vol. 38, no. 4, pp. 740-746, 2020, Epub 2019 Nov 11. PMID: 31692048.

[13] G. Zou, M. Zheng, W. Chen, X. He, and D. Cang, "Autologous platelet-rich plasma therapy for refractory pain after lowgrade medial collateral ligament injury," Journal of International Medical Research, Article ID 300060520903636, 2020, vol. 48, no. 2, PMID: 32090668; PMCID: PMC7111026.

[14] O. N. Özyalvaç, T. Tüzüner, T. Gürpinar, A. Obut, B. Acar, and Y. E. Akman, "Radiological and functional outcomes of ultrasound-guided PRP injections in intrasubstance meniscal degenerations," Journal of Orthopaedic Surgery, Article ID 2309499019852779, 2019, vol. 27, no. 2, PMID: 31204581.

[15] R. Papalia, L. Diaz Balzani, G. Torre et al., "Intraoperative application Platelet rich fibrin, postoperative injections OF PRP or microfracture only for osteochondral lesions of the knee: a five-year retrospective evaluation," Journal of Biological Regulators and Homeostatic Agents, vol. 30, no. 4 Suppl 1, pp. 41-49, 2016, PMID: 28002899.

[16] M. Kemmochi, S. Sasaki, M. Takahashi, T. Nishimura, C. Aizawa, and J. Kikuchi, "The use of platelet-rich fibrin with platelet-rich plasma support meniscal repair surgery," Journal of Orthopaedics, vol. 15, no. 2, pp. 711-720, 2018, Erratum in: J Orthop. 2020 Dec 15;24:293. PMID: 29881226; PMCID: PMC5990248.

[17] M. Shahid and R. Kundra, "Platelet-rich plasma (PRP) for knee disorders," EFORT Open Reviews, vol. 2, no. 2, pp. 28-34, 2017, PMID: 28607768; PMCID: PMC5444079.

[18] S. A. Raeissadat, E. Ghorbani, M. Sanei Taheri et al., "MRI changes after platelet rich plasma injection in knee osteoarthritis (randomized clinical trial)," Journal of Pain Research, vol. 13, pp. 65-73, 2020, PMID: 32021396; PMCID: PMC6959502.
[19] G. Filardo, B. Di Matteo, A. Di Martino et al., "Platelet-rich plasma intra-articular knee injections show no superiority versus viscosupplementation," The American Journal of Sports Medicine, vol. 43, no. 7, pp. 1575-1582, 2015, Epub 2015 May 7. PMID: 25952818.

[20] H. Thermann, R. Fischer, N. Gougoulias, L. Cipollaro, and N. Maffulli, "Endoscopic debridement for non-insertional Achilles tendinopathy with and without platelet-rich plasma," Journal of Sport and Health Science, vol. 30, pp. S2095S2546(20)30078-8, 2020, Epub ahead of print. PMID: 32619656 . 